\title{
A Model for Mobile Social Network Growth
}

\author{
Sreedhar Bhukya \\ Department of Computer Science and Engineering, \\ Sreenidhi Institute of Science and Technology, \\ Yamnampet, Ghatkesar, Hyderabad, India
}

\begin{abstract}
With the recent development in mobile networking technology and increased data speeds mobile phones become a platform for social networking. A serverless friend of friend detection algorithm has been proposed by Macro von Arb et al. Which is implemented successfully on VENETA a mobile social networking platform? Here have studied it more analytically and provided information about vertex degree distribution and clustering coefficient which are the fundamentals aspects of any social network analysis.
\end{abstract}

\section{Keywords}

Mobile Social Networking, Initial contact, Secondary contact. VENETA, Serverless, Friend of friend Detection, Vertex degree distribution.

\section{INTRODUCTION}

With the $3 \mathrm{G}$ technology in the mobile networking data transfer speed has been increased and mobile phones are being used not only for phone calls and SMS but also for data storage and data transfer and also for social networking. Now a days so many mobile based social networking system has been developed like location based mobile social networking, particularly mobile social networking etc. some of such systems are MoSoSo, VENETA, MamJam, Rumble, Plazes, Proxy Dating, Jambo etc. out of which VENETA and Proxy Dating etc. has the advantage that they decompile mobile phone system from the network provider i.e. they don't use any GPRS based internet protocol technique but use only short range blue tooth connectivity technique which is free of cost. Here VENETA has one more advantage that it could implement the friend of friend detection algorithm.

This friend of friend detection in mobile system finds their necessity in most general day to day life activity. For example if we go to any new place where we don't know any baby, we have to switch on our blue tooth Search for our friend of friend instead of enquiring all our friends by making calls. This is very useful in the case of medical emergency and other emergences to keep any help. Another major advantage of this technology in the utilization of mobile coupons. If we have to redeem a mobile coupon which we does not need at that moment, we will search for a friend of friend or friend of friend in our vicinity instead of giving it to a stranger or getting it wasted. In such cases this friend of friend detection plays its major role.

Here we have analyzed thoroughly the serverless friend of friend detection algorithm in mobile social networking [1] which was implemented on VENETA and provided some more detailed and fundamental building blocks of social networking namely the vertex degree distribution and clustering coefficient. Hear we took the help of Ritta Toivonen etal. Proposed "A model for social networks"[4]. Finally we have simulated our proposed model and presented the results.
The interest in calculating this vertex degree distribution and clustering coefficient came from the proposed of ilife; A novel mobile social networks services by Ting Sun et al [3]. Which deals with searching on mobile for a verity of life information like food, clothing, shelter, transportation etc. which id location based and an additional social networking service.

Somehow if one could attach the information about the degree of vertex i.e. number of friends that a person has and also their interests and hobbies to above mentioned friend of friend detection algorithm [3], one could easily find out most influenced person in his/her vicinity who has the interests and hobbies as himself/herself. Finally we have presented simulation results showing the numerical data on the change in degree of vertex (ie. number of contacts of person) and change in number of triangles (ie. change in cluster of people which similar interests) with the introduction of friend of friend detection and friend of friend of friend also possible to detect [12] using mobile.

\section{NETWORK GROWTH ALGORITHM}

The algorithm proposed by Marco von Arb et al. [1]. Is as fallows, Alice (A) owns a set $X=x_{1} \ldots . . x_{N} \subset V$ and Bob (B) owns a Set $Y=y_{1} \ldots . . y_{M} \subset V$. In our case, $\mathrm{X}$ and $\mathrm{Y}$ correspond to the phone numbers in Alice's and Bob's contact books, respectively, and $\mathrm{V}$ is the set of all $10^{7}$ possible 7degited phone numbers. The following protocol allows finding $X \cap Y$.

1) $A \rightarrow B: E_{\alpha}\left(x_{1}\right), \ldots \ldots \ldots E_{\alpha}\left(x_{N}\right)$ ( $\alpha$ Randomly chosen)

2) $B \rightarrow A: E_{\beta}\left(y_{1}\right), \ldots \ldots \ldots E_{\beta}\left(y_{M}\right)$ ( $\beta$ Randomly chosen)

3) $A \rightarrow B: E_{\alpha}\left(E_{\beta}\left(y_{1}\right)\right), \ldots \ldots . ., E_{\alpha}\left(E_{\beta}\left(y_{M}\right)\right)$

4) $B \rightarrow A: E_{\beta}\left(E_{\alpha}\left(x_{1}\right)\right), \ldots \ldots . ., E_{\beta}\left(E_{\alpha}\left(x_{N}\right)\right)$

5) Both, A and B, can compare the lists from step 3 and 4. Due to the commutatively assumption, If $x_{i}=y_{i}$ then $E_{\beta}\left(E_{\alpha}\left(x_{i}\right)\right)=E_{\alpha}\left(E_{\beta}\left(y_{j}\right)\right)$ 


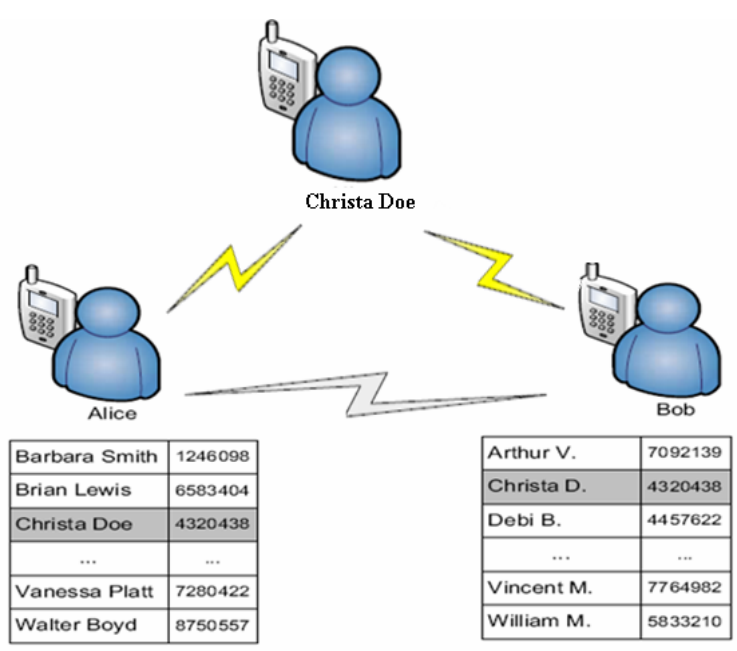

Figure.1 Detecting friends of friends [1]: Whenever the mobile phones of two strangers come into connection range, they can compare their users' contact books. If a matching entry is found, the two strangers are informed to be friends of friends. A possible social network that reflects the information from the contact books, here Alice and Bob are detecting friend of friend and they got Crista Deo is a common friend based on his mobile number and both stringers added their contact books as friend.

Keeping this algorithm in general terms let $\mathrm{m}_{\mathrm{r}} \geq$ number of persons in our contacts list (of $\mathrm{N}$ persons) each found to have $\mathrm{m}_{\mathrm{s}} \geq 0$ number of other friends who are detected to be in our vicinity them we can choose any one of those friend of friend as our friends. Hence our algorithm starts as fallows [4].

1. Start with a seed network of $\mathrm{N}$ vertices

2. Connect an average $m_{r} \geq 1$ initial contact

3. Randomly search an average $m_{s} \geq 0$ neighbors of each initial contact as secondary contact (two strangers know each other )

4. Connect the edge between two strangers as secondary contacts

5. Repeat steps $2-4$ until the network has grown to desired Size (fig.2)

The analytical results are as fallows.

\section{VERTEX DEGREE DISTRIBUTION}

The rate equation which describes how the degree of a vertex changes on average during one time step of the network growth is constructed. The degree $k_{i}$ of vertex $v_{i}$ grows in 2 process [4].

1) When a new vertex directly links to $v_{i}$. Since at any time $\mathrm{t}$, there will be on average $\sim \mathrm{t}$ vertices. Here we are selecting $\mathrm{m}_{\mathrm{r}}$ out of them with a probability $\mathrm{m}_{\mathrm{r}} / \mathrm{t}$.

2) When a vertex links to $v_{i}$ as secondary contact, the selection will give raise to implicit preferential attachment. These will be $\mathrm{m}_{\mathrm{r}} \cdot \mathrm{m}_{\mathrm{s}}$ in number.

These two processes leads to the following rate equation for the degree of vertex $v_{i}[4]$

$$
\frac{\partial \mathrm{k}_{\mathrm{i}}}{\partial \mathrm{t}}=\mathrm{m}_{\mathrm{r}}\left(\frac{1}{\mathrm{t}}+\mathrm{m}_{\mathrm{s}} \frac{\mathrm{k}_{\mathrm{i}}}{\sum \mathrm{k}}\right)=\frac{1}{\mathrm{t}}\left(\mathrm{m}_{\mathrm{r}}+\frac{\mathrm{m}_{\mathrm{s}}}{2\left(1+\mathrm{m}_{\mathrm{s}}\right)} \mathrm{k}_{\mathrm{i}}\right)
$$

From this the probability density distribution for the degree $\mathrm{k}$ is given by

$$
P(k)=A B^{A}(k+C)^{-2 / m_{s}+2 m_{s} m_{t}-3}
$$

Where

$$
A=2\left(1+m_{s}\right) / m_{s}, B=m_{r}\left(A+1+m_{s}\right), C=A m_{r}
$$

\section{CLUSTERING SPECTRUM}

The clustering coefficient on vertex degree can also be found by the rate equation method [5-11], let us examine how the number of triangles $E_{i}$ changes $v_{i}$ changes with time. The triangle around $\mathrm{v}_{\mathrm{i}}$ are mainly generated by two process [4]

1. Vertex $\mathrm{v}_{\mathrm{i}}$ is chosen as one of the initial contact with probability $\mathrm{m}_{\mathrm{r}} / \mathrm{t}$

2. New vertex links to some of its neighbors, giving raise to triangles

These two processes are describes by the rate equation

$$
\frac{\partial \mathrm{E}_{\mathrm{i}}\left(\mathrm{k}_{\mathrm{i}}, \mathrm{t}\right)}{\partial \mathrm{t}}=\frac{\mathrm{m}_{\mathrm{r}} \mathrm{m}_{\mathrm{s}}}{\mathrm{t}}+\mathrm{m}_{\mathrm{r}} \mathrm{m}_{\mathrm{s}} \frac{\mathrm{k}_{\mathrm{i}}}{\sum \mathrm{k}}=\frac{\partial \mathrm{k}_{\mathrm{i}}}{\partial \mathrm{t}}+\frac{\mathrm{m}_{\mathrm{r}}\left(\mathrm{m}_{\mathrm{s}}-1\right)}{\mathrm{t}}
$$

We get the time evaluation of triangles around a vertex $v_{i}$ as

$$
E_{i}(t)=k_{i}(t)+m_{r}\left(m_{s}-1\right) \ln \left(\frac{t}{t_{i}}\right)-m_{r}
$$

From this we can obtain the clustering the coefficient as [2]

$$
c_{i}\left(k_{i}\right)=\frac{2 E_{i}\left(k_{i}\right)}{k_{i}\left(k_{i}-1\right)}=2 \frac{k_{i}+D \ln \left(k_{i}+C\right)-F}{k_{i}\left(k_{i}-1\right)}
$$

Where $\mathrm{C}=\mathrm{Am} \mathrm{m}_{\mathrm{r}}, \mathrm{D}=\mathrm{C}\left(\mathrm{m}_{\mathrm{s}}-\mathbf{1}\right), \mathrm{F}=\mathrm{D} \ln \mathrm{B}+\mathrm{m}_{\mathrm{r}}$ For large values of degree $\mathrm{k}$, the clustering coefficient thus depends on $\mathrm{k}$ as $\mathrm{c}(\mathrm{k}) \sim 1 / \mathrm{k}$.

\section{SIMULATION RESULTS}

In the simulation part we have developed a model network with 50 nodes (Fig.1) and calculated the sum of total number of initial and secondary contacts of each person and in the table (1) we have presented a ratio of sum of total number of initial contacts to the total number of nodes (50) and that of secondary contacts to the total number of nodes.

Comparing to the case of triangle formation, a triangle if formed in the network if one makes a friend of friend as his/her friend. This triangle is formed via a secondary contact. Also there is a possibility of triangle formation between three initial contacts ie. There occurs a possibility that two persons 
in or contacts list be friend which may be unknown to us. This possibility of new triangle formation is very less as compared to the triangle formation by making friend of friend as friend figure. 2 describes the vertex degree distribution with number of nodes as the network grows. Here blue indicates the degree due to initial contacts, red line indicates the degree due to secondary contacts and yellow line indicates total degree of nodes. This clearly shows the influence of secondary contact (ie. making friend of friend as friend) on the degree of vertex.

Mobile social network graph

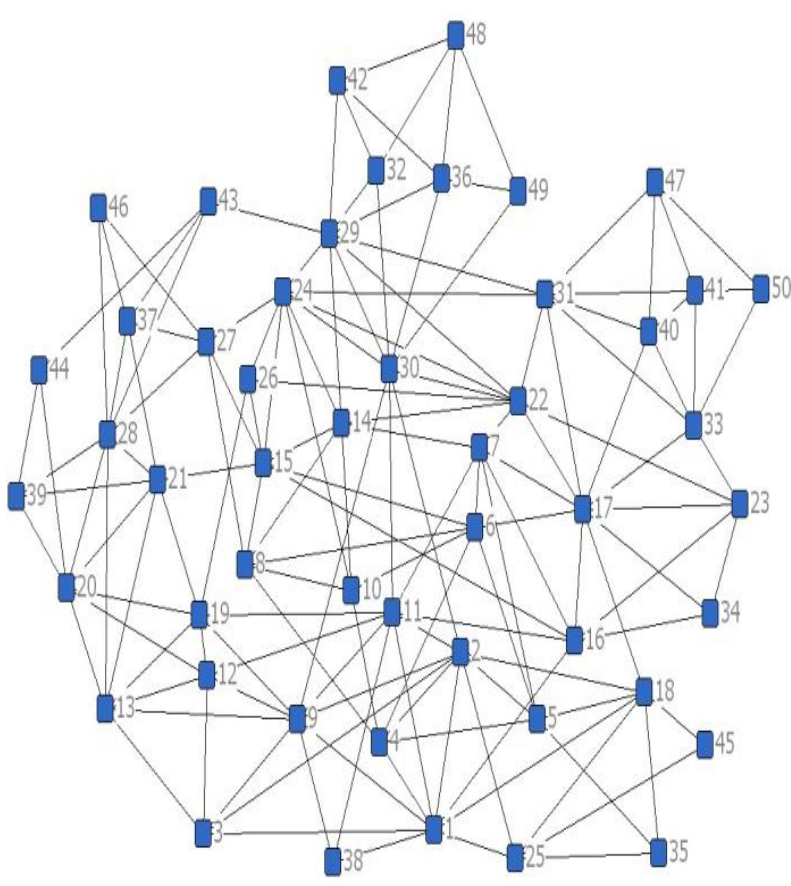

Fig. 2

Figure4 depicts the total degree distribution as function of number of vertex. From the table and plots one can easily observe an enormous growth in the degree of vertex and number of triangles due to introduction of friend of friend as secondary contact.

Table.1

\begin{tabular}{|c||c|c|c|}
\hline & $\begin{array}{c}\text { Initial } \\
\text { Friend } \\
(\mathrm{IC})\end{array}$ & $\begin{array}{c}\text { Secondary } \\
\text { Contacts } \\
(\mathrm{SC})\end{array}$ & Difference \\
\hline Vertices & 2.28 & 3.48 & 1.2 \\
\hline Triangles & 0.2 & $5.34 \%$ & 5.14 \\
\hline
\end{tabular}

The growth in the mobile social network is more using secondary contact compared to that using initial contact.

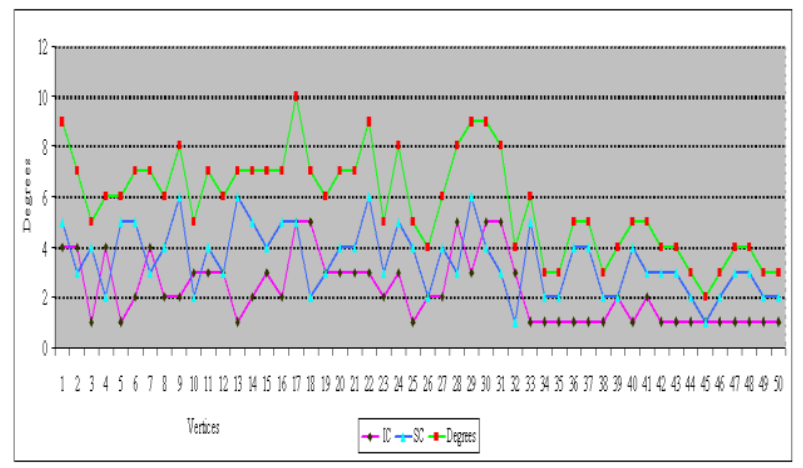

Fig.3 display of compared results of growing network community, initial contacts are growing at a very slow rate compared to secondary contact i.e. indicates the initial contact, $\Delta$ indicates secondary contacts and $n$ indicates degree of each vertices, when initial and secondary contact connect to a vertex $\mathrm{v}_{\mathrm{i}}$ are considered.

\begin{tabular}{|c|l|}
\hline Notation & Description \\
\hline $\mathrm{m}_{\mathrm{r}}$ & Random Initial Contact \\
\hline $\mathrm{m}_{\mathrm{s}}$ & Secondary Contact \\
\hline
\end{tabular}

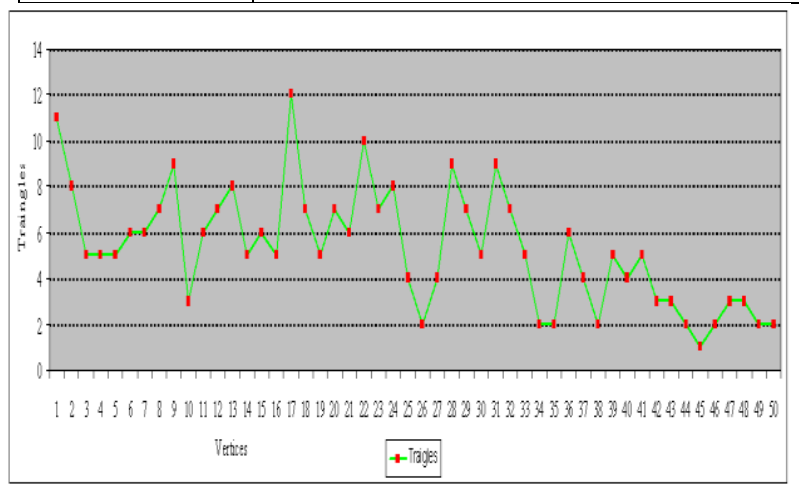

Fig. 4 a indicates triangles of each vertex, when the two strangers are connecting it forms a triangle, growth of triangles using secondary contacts.

\section{Tools}

We have used UciNet, NetDraw and Excel for creating graph and simulation results, detects of friends of friends is shown in VENETA (www.veneta-project.net).

\section{CONCLUSION}

We have developed a mobile social network model which satisfies assortative mixing, high clustering, short average path length, broad degree distribution and the existence of mobile community structure. Using secondary contact we have shown by simulation results growth of mobile social network is more using secondary contact compared to that using initial contact. This model, as mentioned in the introduction, hopefully can be implemented on VENETA as an execution to the model proposed by Marco van Arb [1] which is not work service provides independent and works via blue tooth which intern competes which the ilife; model 
proposed by Ting Sun etal. Which is a mobile internet based service.

\section{Notations}

\section{REFERENCES}

[1] Marco von Arb, Matthias Bader, Michael Kuhn and Roger Wattenhofer. VENETA: Serverless Friend-ofFriend Detection in Mobile Social Networking. In IEEE International Conference on Wireless \& Mobile Computing, Networking \& Communication, 2008.

[2] J. Clerk Maxwell, A Treatise on Electricity and Magnetism, 3rd ed., vol. 2. Oxford: Clarendon, 1892, pp.68-73.

[3] Ting Sun, Xiaoyu Shi, Yanming Shen, Keqiu Li, Wenyu Qu "ilife: A novel mobile social network services on mobile phone" IEEE, CIT 2010.

[4] Riitta Toivonen, Jukka-Pekka Onnela, Jari Sarama ki, Jo"rkki Hyvo"nen, Kimmo Kaski "A model for social networks". Physica A 371 (2006) 851-860

[5] Flora S. Tsai , Wenchou Han, Junwei Xu, Hock Chuan Chua "Design and development of a mobile peer-to-peer social networking application" Expert Systems with Applications 36 (2009) 11077-11087

[6] M. J. Freedman, K. Nissim, and B. Pinkas. Efficient Private Matching and Set Intersection. In EUROCRYPT, pages 1-19, 2004.

[7] S. Hohenberger and S. A. Weis. Honest-Verifier Private Disjointness Testing Without Random Oracles. In Privacy Enhancing Technologies, pages 277-294, 2006

[8] A. Kiayias and A. Mitrofanova. Testing Disjointness of Private Datasets. In Financial Cryptography, pages 109 124, 2005.

[9] P. Krapivsky, S. Redner, Phys. Rev. E 63 (2001) 066123.

[10] S. Dorogovtsev, J. Mendes, A. Samukhin, Phys. Rev. Lett. 85 (2000) 4633.

[11] G. Szabo’, M. Alava, J. Kerte’sz, Phys. Rev. E 67 (2003) 056102.

[12] Sreedhar Bhukya, A novel model for social network, BCFIC, Pages. 21-24, IEEE, 2011. 